\title{
A THEORETICAL DETERMINATION OF THE RATE CONSTANT FOR OH HYDROGEN ABSTRACTION FROM TOLUENE
}

Víctor H. Uc ${ }^{1}$, J. Raúl Alvarez-Idaboy ${ }^{2}$, Annia Galano${ }^{1}$, Isidoro García-Cruz ${ }^{1}$, Annik Vivier-Bunge ${ }^{3}$

${ }^{1}$ Instituto Mexicano del Petróleo, Eje Central Lázaro Cardenas 152, 07730, México D.F.

${ }^{2}$ Facultad de Química, Universidad Nacional Autónoma de México, Ciudad Universitaria, 04510 México D.F., México

${ }^{3}$ Universidad Autónoma Metropolitana, Unidad Iztapalapa, Departamento de Química, Área de Química Cuántica C.P. 09340 México D.F.

\section{Supplementary Material}


Table 1: Electronic energies and ZPE corrections calculated at the BHandHLYP/6-31++G(d,p)level, and $\operatorname{CCSD}(\mathrm{T}) / 6-31++\mathrm{G}(\mathrm{d}, \mathrm{p}) / / \mathrm{BHandHLYP} / 6-31++\mathrm{G}(\mathrm{d}, \mathrm{p})$ electronic energies, for all channels of the toluene $+\mathrm{OH}$ abstraction reaction, in hartrees.

\begin{tabular}{|c|c|c|c|}
\hline & BHandHLYP $/ 6-311++\mathrm{g}^{* *}$ & $\mathrm{ZPE}$ & $\mathrm{CCSD}(\mathrm{T}) / 6-311++\mathrm{g}^{* *}$ \\
\hline $\mathrm{OH}$ & -75.7296238 & 0.008846 & -75.5963818 \\
Tolueno & -271.4590038 & 0.131703 & -270.8747813 \\
$\mathrm{H}_{2} \mathrm{O}$ & -76.4144083 & 0.02215 & -76.286343 \\
\hline Methyl & & & \\
TS & -347.1809283 & 0.138053 & -346.4646264 \\
Product & -270.8122856 & 0.117808 & -270.221782 \\
\hline Ortho & & & \\
TS & -347.1707536 & 0.137143 & -346.4576727 \\
Product & -270.7746275 & 0.118466 & -270.1885246 \\
\hline Meta & & & \\
TS & -347.1702983 & 0.136891 & -346.4568784 \\
Product & -270.7742425 & 0.118284 & -270.1884951 \\
\hline Para & & & \\
TS & -347.1699345 & 0.136889 & -346.4562464 \\
Product & -270.7733742 & 0.11831 & -270.1876982 \\
\hline
\end{tabular}


Table 2: Cartesian coordinates of toluene at the BHandHLYP/6-31++G(d,p) level.

\begin{tabular}{|c|c|c|c|}
\hline \multirow{2}{*}{ Atomic } & \multicolumn{3}{|c|}{ Coordinates (Angstroms) } \\
\cline { 2 - 4 } Number & $\mathrm{X}$ & $\mathrm{Y}$ & $\mathrm{Z}$ \\
\hline 6 & -1.194965 & -0.003949 & -1.191352 \\
6 & -1.192200 & 0.009962 & 0.192808 \\
6 & -0.000047 & 0.012895 & 0.905809 \\
6 & 1.192189 & 0.008351 & 0.192911 \\
6 & 1.195068 & -0.005564 & -1.191228 \\
6 & 0.000071 & -0.012562 & -1.889575 \\
1 & -2.130726 & -0.004258 & -1.724025 \\
1 & -2.128209 & 0.020693 & 0.726919 \\
1 & 2.128153 & 0.017818 & 0.727127 \\
1 & 2.130869 & -0.007137 & -1.723829 \\
1 & 0.000127 & -0.020475 & -2.965869 \\
6 & -0.000098 & -0.003610 & 2.407341 \\
1 & -0.000163 & -1.023925 & 2.784565 \\
1 & 0.878280 & 0.492025 & 2.807443 \\
1 & -0.878441 & 0.492126 & 2.807387 \\
\hline
\end{tabular}

Table 3: Cartesian coordinates of $\mathrm{OH}$ at the BHandHLYP/6-31++G(d,p) level.

\begin{tabular}{|c|c|c|c|}
\hline \multirow{2}{*}{$\begin{array}{c}\text { Atomic } \\
\text { Number }\end{array}$} & \multicolumn{3}{|c|}{ Coordinates (Angstroms) } \\
\cline { 2 - 4 } & $\mathrm{X}$ & $\mathrm{Y}$ & $\mathrm{Z}$ \\
\hline 8 & 0.000000 & 0.000000 & -0.106992 \\
1 & 0.000000 & 0.000000 & 0.855939 \\
\hline
\end{tabular}


Table 4: Cartesian coordinates of TSs at the BHandHLYP/6-31++G(d,p) level.

\begin{tabular}{|c|c|c|c|}
\hline Atom & $\mathrm{X}$ & $\mathrm{Y}$ & $\mathrm{Z}$ \\
\hline 6 & -0.389693 & 0.383923 & -0.233079 \\
6 & -0.390081 & 0.398184 & 1.160310 \\
6 & 0.796542 & 0.421141 & 1.869377 \\
6 & 2.008383 & 0.430699 & 1.197731 \\
6 & 2.023944 & 0.417855 & -0.187676 \\
6 & 0.836381 & 0.394900 & -0.895166 \\
1 & -1.329345 & 0.393448 & 1.688179 \\
1 & 0.776667 & 0.435593 & 2.945646 \\
1 & 2.932512 & 0.450971 & 1.749108 \\
1 & 2.961686 & 0.429742 & -0.716313 \\
1 & 0.854866 & 0.387603 & -1.972426 \\
6 & -1.657443 & 0.331695 & -0.989442 \\
1 & -1.590392 & 0.736762 & -1.991236 \\
1 & -1.982005 & -0.804468 & -1.181334 \\
1 & -2.506668 & 0.739152 & -0.455623 \\
8 & -2.250896 & -2.122842 & -1.339939 \\
1 & -1.478346 & -2.456439 & -0.878823 \\
\hline
\end{tabular}


Table 5: Cartesian coordinates of $\mathrm{TSr}_{o}$ at the BHandHLYP/6-31++G(d,p) level.

\begin{tabular}{|c|c|c|c|}
\hline \multirow{2}{*}{ Atomic } & \multicolumn{3}{|c|}{ Coordinates (Angstroms) } \\
\cline { 2 - 4 } Number & $\mathrm{X}$ & $\mathrm{Y}$ & $\mathrm{Z}$ \\
\hline 6 & -1.542948 & 0.007897 & -1.515468 \\
6 & -1.566448 & 0.031443 & -0.129358 \\
6 & -0.365009 & 0.044933 & 0.540749 \\
6 & 0.871095 & 0.031234 & -0.071901 \\
6 & 0.862255 & 0.012534 & -1.465426 \\
6 & -0.325173 & -0.000965 & -2.176057 \\
1 & -2.464984 & 0.002398 & -2.070871 \\
1 & -2.497527 & 0.048335 & 0.409891 \\
1 & -0.403357 & 0.123311 & 1.801907 \\
1 & 1.801398 & 0.011451 & -1.994745 \\
1 & -0.299868 & -0.014904 & -3.252254 \\
6 & 2.155052 & 0.023445 & 0.705568 \\
1 & 2.913252 & 0.617251 & 0.205967 \\
1 & 2.009077 & 0.415488 & 1.704602 \\
1 & 2.540678 & -0.989074 & 0.799807 \\
1 & -0.542215 & -0.969845 & 3.061240 \\
8 & -0.448675 & -0.018441 & 3.000727 \\
\hline
\end{tabular}


Table 6: Cartesian coordinates of $\mathrm{TSr}_{m}$ at the BHandHLYP/6-31++G(d,p) level.

\begin{tabular}{|c|c|c|c|}
\hline \multirow{2}{*}{ Atomic } & \multicolumn{3}{|c|}{ Coordinates (Angstroms) } \\
\cline { 2 - 4 } Number & $\mathrm{X}$ & $\mathrm{Y}$ & $\mathrm{Z}$ \\
\hline 6 & 0.000000 & 0.000000 & 0.000000 \\
6 & 0.000000 & 0.000000 & 1.387262 \\
6 & 1.221795 & 0.000000 & 2.017797 \\
6 & 2.422641 & -0.008290 & 1.350068 \\
6 & 2.420212 & -0.004065 & -0.043041 \\
6 & 1.195397 & -0.004031 & -0.699616 \\
1 & -0.934521 & 0.000206 & -0.535122 \\
1 & -0.918877 & 0.003934 & 1.946279 \\
1 & 1.247121 & 0.038744 & 3.282290 \\
1 & 1.240230 & -1.116399 & 4.489736 \\
8 & 1.273979 & -0.159357 & 4.469199 \\
1 & 3.352246 & -0.012157 & 1.893911 \\
1 & 1.176461 & -0.008435 & -1.776872 \\
6 & 3.711242 & 0.025972 & -0.809623 \\
1 & 4.064152 & 1.047391 & -0.931443 \\
1 & 4.488487 & -0.529025 & -0.294934 \\
1 & 3.590241 & -0.400191 & -1.799647 \\
\hline
\end{tabular}


Table 7: Cartesian coordinates of $\operatorname{TSr}_{p}$ at the BHandHLYP/6-31++G(d,p) level.

\begin{tabular}{|c|c|c|c|}
\hline \multirow{2}{*}{$\begin{array}{c}\text { Atomic } \\
\text { Number }\end{array}$} & \multicolumn{3}{|c|}{ Coordinates (Angstroms) } \\
\cline { 2 - 4 } & $\mathrm{X}$ & $\mathrm{Y}$ & $\mathrm{Z}$ \\
\hline 6 & 0.000000 & 0.000000 & 0.000000 \\
6 & 0.000000 & 0.000000 & 1.387377 \\
6 & 1.212242 & 0.000000 & 2.035408 \\
6 & 2.413680 & -0.009315 & 1.367633 \\
6 & 2.390987 & -0.009234 & -0.019558 \\
6 & 1.189706 & -0.001587 & -0.718774 \\
1 & -0.939695 & 0.000476 & -0.527808 \\
1 & -0.924536 & 0.002699 & 1.937859 \\
1 & 1.222824 & 0.045740 & 3.302197 \\
1 & 3.321898 & -0.015984 & -0.562667 \\
1 & 1.227546 & -1.104347 & 4.511104 \\
8 & 1.231889 & -0.146896 & 4.486476 \\
6 & 1.177536 & 0.030156 & -2.220656 \\
1 & 1.178216 & 1.054640 & -2.585667 \\
1 & 2.050703 & -0.464413 & -2.632583 \\
1 & 0.294226 & -0.458125 & -2.618213 \\
1 & 3.347088 & -0.013792 & 1.902925 \\
\hline
\end{tabular}


Table 8: Cartesian coordinates of product in ortho at the BHandHLYP/6-31++G(d,p) level.

\begin{tabular}{|c|c|c|c|}
\hline \multirow{2}{*}{$\begin{array}{c}\text { Atomic } \\
\text { Number }\end{array}$} & \multicolumn{3}{|c|}{ Coordinates (Angstroms) } \\
\cline { 2 - 4 } & $\mathrm{X}$ & $\mathrm{Y}$ & $\mathrm{Z}$ \\
\hline 6 & 0.000000 & 0.000000 & 0.000000 \\
6 & 0.000000 & 0.000000 & 1.392860 \\
6 & 1.218829 & 0.000000 & 2.006883 \\
6 & 2.449252 & 0.000123 & 1.396865 \\
6 & 2.409548 & 0.000206 & 0.003399 \\
6 & 1.203691 & 0.000131 & -0.683121 \\
1 & -0.932557 & -0.000035 & -0.538494 \\
1 & -0.920798 & -0.000121 & 1.950604 \\
1 & 3.336828 & 0.000291 & -0.546944 \\
1 & 1.207577 & 0.000182 & -1.759556 \\
6 & 3.738644 & 0.000174 & 2.164963 \\
1 & 4.589370 & 0.000585 & 1.492725 \\
1 & 3.810811 & 0.875453 & 2.803507 \\
1 & 3.811225 & -0.875430 & 2.803003 \\
\hline
\end{tabular}


Table 9: Cartesian coordinates of product in meta at the BHandHLYP/6-31++G(d,p) level.

\begin{tabular}{|c|c|c|c|}
\hline \multirow{2}{*}{$\begin{array}{c}\text { Atomic } \\
\text { Number }\end{array}$} & \multicolumn{3}{|c|}{ Coordinates (Angstroms) } \\
\cline { 2 - 4 } & $\mathrm{X}$ & $\mathrm{Y}$ & $\mathrm{Z}$ \\
\hline 6 & 0.000000 & 0.000000 & 0.000000 \\
6 & 0.000000 & 0.000000 & 1.390852 \\
6 & 1.229732 & 0.000000 & 1.987659 \\
6 & 2.434695 & -0.001314 & 1.344988 \\
6 & 2.423012 & 0.003147 & -0.051772 \\
6 & 1.194414 & 0.000497 & -0.702988 \\
1 & -0.935578 & -0.003626 & -0.534292 \\
1 & -0.917065 & -0.002308 & 1.953500 \\
1 & 3.366396 & -0.005757 & 1.886157 \\
1 & 1.171391 & -0.002911 & -1.780023 \\
6 & 3.709961 & 0.036243 & -0.825610 \\
1 & 4.061033 & 1.058335 & -0.947633 \\
1 & 4.490350 & -0.518979 & -0.315852 \\
1 & 3.584282 & -0.388521 & -1.815719 \\
\hline
\end{tabular}


Table 10: Cartesian coordinates of product in para at the BHandHLYP/6-31++G(d,p) level.

\begin{tabular}{|c|c|c|c|}
\hline \multirow{2}{*}{$\begin{array}{c}\text { Atomic } \\
\text { Number }\end{array}$} & \multicolumn{3}{|c|}{ Coordinates (Angstroms) } \\
\cline { 2 - 4 } & $\mathrm{X}$ & $\mathrm{Y}$ & $\mathrm{Z}$ \\
\hline 6 & 0.000000 & 0.000000 & 0.000000 \\
6 & 0.000000 & 0.000000 & 1.391115 \\
6 & 1.219975 & 0.000000 & 2.006781 \\
6 & 2.425537 & -0.004139 & 1.363369 \\
6 & 2.393713 & -0.004088 & -0.027385 \\
6 & 1.188767 & 0.001319 & -0.721398 \\
1 & -0.940589 & -0.002865 & -0.527147 \\
1 & -0.922966 & -0.002399 & 1.944937 \\
1 & 3.321981 & -0.010144 & -0.575911 \\
6 & 1.171625 & 0.033758 & -2.224053 \\
1 & 1.168881 & 1.057403 & -2.591634 \\
1 & 2.044018 & -0.459986 & -2.638903 \\
1 & 0.288562 & -0.457478 & -2.618825 \\
1 & 3.360918 & -0.009712 & 1.895931 \\
\hline
\end{tabular}


Table 11: Cartesian coordinates of benzyl at the BHandHLYP/6-31++G(d,p) level.

\begin{tabular}{|c|c|c|c|}
\hline \multirow{2}{*}{$\begin{array}{c}\text { Atomic } \\
\text { Number }\end{array}$} & \multicolumn{3}{|c|}{ Coordinates (Angstroms) } \\
\cline { 2 - 4 } & $\mathrm{X}$ & $\mathrm{Y}$ & $\mathrm{Z}$ \\
\hline 6 & 0.000000 & 0.000000 & 0.000000 \\
6 & 0.000000 & 0.000000 & 1.392056 \\
6 & 1.210343 & 0.000000 & -0.687673 \\
1 & -0.929909 & 0.000000 & -0.541167 \\
6 & 1.185957 & 0.000000 & 2.089277 \\
6 & 2.402384 & 0.000000 & -0.000905 \\
1 & -0.934312 & 0.000000 & 1.927120 \\
1 & 1.214044 & 0.000000 & -1.764340 \\
6 & 2.429410 & 0.000000 & 1.413871 \\
1 & 1.179087 & 0.000000 & 3.166236 \\
1 & 3.335318 & 0.000000 & -0.538990 \\
6 & 3.639506 & 0.000000 & 2.118222 \\
1 & 3.654397 & 0.000000 & 3.193300 \\
1 & 4.581758 & 0.000000 & 1.600367 \\
\hline
\end{tabular}

Table 12: Cartesian coordinates of product $\mathrm{H}_{2} \mathrm{O}$ at the BHandHLYP $/ 6-31++\mathrm{G}(\mathrm{d}, \mathrm{p})$ level.

\begin{tabular}{|c|c|c|c|}
\hline \multirow{2}{*}{ Atomic } & \multicolumn{3}{|c|}{ Coordinates (Angstroms) } \\
\cline { 2 - 4 } Number & $\mathrm{X}$ & $\mathrm{Y}$ & $\mathrm{Z}$ \\
\hline 1 & 0.000000 & 0.000000 & 0.000000 \\
8 & 0.000000 & 0.000000 & 0.950594 \\
1 & 0.915074 & 0.000000 & 1.208018 \\
\hline
\end{tabular}

